\title{
A novel missense mutation of the tissue-nonspecific alkaline phosphatase gene detected in a patient with hypophosphatasia
}

\begin{abstract}
Hypophosphatasia is a rare heritable inborn error of metabolism characterized by abnormal bone mineralization associated with a deficiency of alkaline phosphatase. The clinical expression of hypophosphatasia is highly variable, ranging from death in utero to pathologic fractures first presenting in adulthood. We investigated the tissuenonspecific alkaline phosphatase (TNSALP) gene from a Japanese female patient with hypophosphatasia. By a quantitative polymerase chain reaction (PCR) method, the amount of TNSALP mRNA appeared to be almost equal to that in normal individuals. Gene analysis clarified that the hypophosphatasia originated from a missense mutation and a nucleotide deletion. The missense mutation, a $\mathrm{C} \rightarrow \mathrm{T}$ transition at position 1041 of cDNA, results in an amino acid change from Leu to Phe at codon 272, which has not yet been reported. The previously reported deletion of $\mathrm{T}$ at 1735 causes a frame shift mutation downstream from Leu at codon 503. Family analysis showed that the mutation 1041T and the deletion $1735 \mathrm{~T}$ had been inherited from the proband's father and mother, respectively. An expression experiment revealed that the mutation 1041T halved the expression of alkaline phosphatase activity. Using homology analysis, the Leu-272 was confirmed to be highly conserved in other mammals.
\end{abstract}

Key words Frameshift mutation - Bone marrow stromal fibroblast · Glycosyl-phosphatidyl-inositol (GPI) · Hepatic failure

N. Sugimoto $(\varangle) \cdot$ Y. Hoshino

Department of Orthopaedics, Jichi Medical School, Minamikawachimachi, Tochigi 329-0498, Japan

Tel. +81-0285-44-2111; Fax +81-0285-44-4902

e-mail: nsugimot@jichi.ac.jp

S. Iwamoto $\cdot$ E. Kajii

Department of Legal Medicine and Human Genetics, Jichi Medical

School, Tochigi, Japan

\section{Introduction}

Alkaline phosphatases (ALPs) [orthophosphoric-monoester phosphohydrolase (alkaline optimum), EC 3.1.3.1] are cell surface glycoproteins that can hydrolyze a variety of monophosphate esters with high $\mathrm{pH}$ optima, and we now know that there are at least four gene loci encoding their protein moieties (McComb et al. 1979). The L/B/K ALP (liver/ bone/ kidney or "tissue non-specific" alkaline phosphatase: TNSALP) is widespread and shows particularly high activity in mineralizing bone, where it is located mainly in the plasma membrane of osteoblastic cells (Harris 1989). TNSALP cDNA was reported by Weiss et al. in 1986. Three other forms of ALP are intestinal ALP (IALP), placental ALP (PALP), and placental-like ALP (PLALP). The TNSALP is located near the end of the short arm of chromosome 1 (p36-p34), while IALP, PALP, and PLALP are closely linked and located near the end of the long arm of chromosome 2 (q34q37) (Smith et al. 1988; Griffin et al. 1987).

Recent studies indicate that PALP and PLALP belong to a group of membrane proteins anchored to the plasma membrane by a $\mathrm{COOH}$-terminal glycosyl-phosphatidylinositol (GPI) structure (Amthauer et al. 1992; Coyne et al. 1993; Kodukula et al. 1993). The structure and biosynthesis of the GPI moiety have been under intensive investigation.

Hypophosphatasia is an inborn error of metabolism characterized by a lack of TSNALP activity. This condition results in defective mineralization of bones and teeth, and, in severe forms of hypophosphatasia, various systemic symptoms also occur. The clinical expression of hypophosphatasia is highly variable, ranging from stillbirth without radiographically apparent mineralized bone to a lack of pathologic fracture development until late in adulthood, but there is a general relationship between the age at onset of symptoms, their severity, and prognosis (Fraser 1957; Currarino et al. 1957).

In this study, we analyzed the TNSALP gene, using bone marrow stromal fibroblasts cultured from a patient with hypophosphatasia, to reveal its mutations, inheritance, and clinical expression. 


\section{Materials and methods}

Patient's case history

The proband was the second child born of unconsanguineous Japanese parents. Two children in this family were affected by hypophosphatasia. The proband had been diagnosed with hypophosphatasia at age 12 days and underwent a spinal operation for kyphosis at age 12 years. Serum ALP levels (normal range, 89-285 IU/1) were 10 IU/1 in the patient, 45IU/1 in her mother, and $89 \mathrm{IU} / 1$ in her father. Although her mother had low serum ALP, she showed no symptoms of hypophosphatasia. At age 16 years, the patient was hospitalized because of an episode of severe convwlsions and liver and renal dysfunction. She died of acute hepatic failure on the 12th day after admission. Her brother's hypophosphatasia was detected at age 3 months. At age 14 years, he died of hepatic failure due to an unknown cause.

\section{Cell preparation}

Bone stromal fibroblasts from the proband were propagated from bone marrow cells, which were taken at the time of her spinal instrumentation operation after informed consent had been obtained.

Isolation of TNSALP cDNA from bone marrow stromal fibroblasts

TNSALP mRNA was isolated from cultured bone marrow stromal fibroblasts using a Total RNA Separator Kit (Clontech Laboratories, Palo Alto, CA, USA), according to the supplier's instructions, and was reverse-transcribed using $\mathrm{d}(\mathrm{T}) 16$ as a primer. This cDNA was then amplified using a GeneAmp XL PCR Kit (Perkin-Elmer/Roche, Branch burg, NJ, USA), with primers produced on the basis of published sequences. The primer set consisted of primer $S$ (5'-TCCAGGGATAAAGCAGGTCT-3': for TNSALP nucleotides positions 146-165) and primer A (5'GGAAGTTGGCATATGTCAAG-3': for TNSALP nucleotides positions 1799-1780) (Weiss et al. 1986). After 30 cycles of amplification using these primers, the reaction products were separated by agarose gel electrophoresis and visualized by ethidium bromide staining.

The polymerase chain reaction (PCR) product was inserted into the plasmid vector pCRII (TA cloning system: Invitrogen, San Diego, CA, USA).

\section{DNA sequence analysis}

The nucleotide sequence was determined by the dideoxy nucleotide chain termination method with the use of a Sequenase DNA sequencing kit (US Biochemical, Cleveland, $\mathrm{OH}, \mathrm{USA})$ and $\left[\alpha^{32} \mathrm{P}\right]$ deoxy cyticine triphosphate (CTP) (Sanger et al. 1977). The reaction products were electrophoresed on $6 \%$ acrylamide gels containing $8 \mathrm{M}$ urea.
Quantitation of the relative amount of TNSALP mRNA

As an internal control, a 723-bp fragment of $\beta$-actin cDNA was amplified by primer- $\beta$-act $\mathrm{S}\left(5^{\prime}-\right.$ ATCATGTTTGAGACCTTCAA-3') and primer- $\beta$-act A (5'-GTCATACTCCTGCTTGCTGA-3'), which have been described previously (Sato et al. 1994). A 437-bp fragment of TNSALP cDNA was amplified by primer $\mathrm{qS}\left(5^{\prime}-\right.$ TCACATTTGGTGGATA-CACC-3') and primer A. As a control, age- and sex-matched mRNA isolated from bone stromal fibroblasts was prepared.

Expression of wild-type and mutant alkaline phosphatase

The wild-type and mutant alkaline phosphatases were digested by EcoRI, and inserted into expression plasmid pCR3-Uni using the Eukaryotic Cloning Kit-Unidirectional (Invitrogen). They were purified using the EndoFree Plasmid Maxi Kit (Qiagen, Chatsworth, CA, USA). NIH/ $3 \mathrm{~T} 3$ cells [a gift from the Health Science Research Resources Bank (Japan Health Sciences Foundation, Tokyo)], were transfected with circular plasmid DNA using Lipofectamine Reagent (Life Technologies, Gaithersburg, MD, USA). Cellular RNAs were isolated using the Total RNA Separator Kit (Clontech Laboratories) and $15 \mu \mathrm{g}$ of each RNA was fractionated in a $1 \%$ agarose gel with formaldehyde, transferred to Hybond-N membrane (Amersham International plc, Buckinghamshire, UK), and hybridized to $\left[\alpha-{ }^{32} \mathrm{P}\right]$-labeled TNSALP cDNA.

\section{Determination of enzyme activity}

Transfected cells were obtained by scraping from a $35-\mathrm{mm}$ culture plate and homogenizing in $1 \mathrm{ml}$ of distilled water. ALP activity was measured by the method of Lowry et al. (1954), and by a modification of the method of The Committee on Enzymes of the Scandinavian Society for Clinical Chemistry and Clinical Physiology (1974). A $10-\mu \mathrm{l}$ aliquot of each cell lysate was added to $100 \mathrm{ml}$ paranitrophenylphosphate solution after pre-heating in the diethanolamine $\mathrm{MgCl}_{2}$ buffer. The tubes were incubated in a water bath at $37^{\circ} \mathrm{C}$ for $0,3,5,10,20$, or $30 \mathrm{~min}$. Each reaction was terminated by the addition of $180 \mu \mathrm{l}$ of $1 \mathrm{~N}$ $\mathrm{NaOH}$, and the absorbance was measured at $405 \mathrm{~nm}$ by a Hitachi U-2000 Spectrophotometer (Hitachi, Hitachinaka, Japan). The ALP activity of each transfected cell was examined microscopically after histochemical staining with Naphthol AS-BI phosphate $\mathrm{C}_{18} \mathrm{H}_{15} \mathrm{Br} \mathrm{NO}_{6} \mathrm{P}$ (N2125, SIGMA, St. Louis, Missouri, USA) and new-fuchsin (Nanba et al. 1987).

\section{Results}

We examined the TNSALP gene of a patient with hypophosphatasia. The TNSALP cDNA was synthesized from bone marrow stromal fibroblasts, and then a 1654-bp segment was amplified using primers that flanked the entire 
Fig. 1A,B Sequence analysis of the normal and the mutant alleles. a Mutant tissue-nonspecific alkaline phosphatase (TNSALP) cDNA demonstrated a $\mathrm{C} \rightarrow \mathrm{T}$ transition at nucleotide position 1041, resulting in an amino acid change of Leu to Phe at codon 272. b The mutant TNSALP cDNA demonstrated deletion of $\mathrm{T}$ at nucleotide position 1735. This deletion causes the frameshift mutation, downstream from the Leu at codon 503

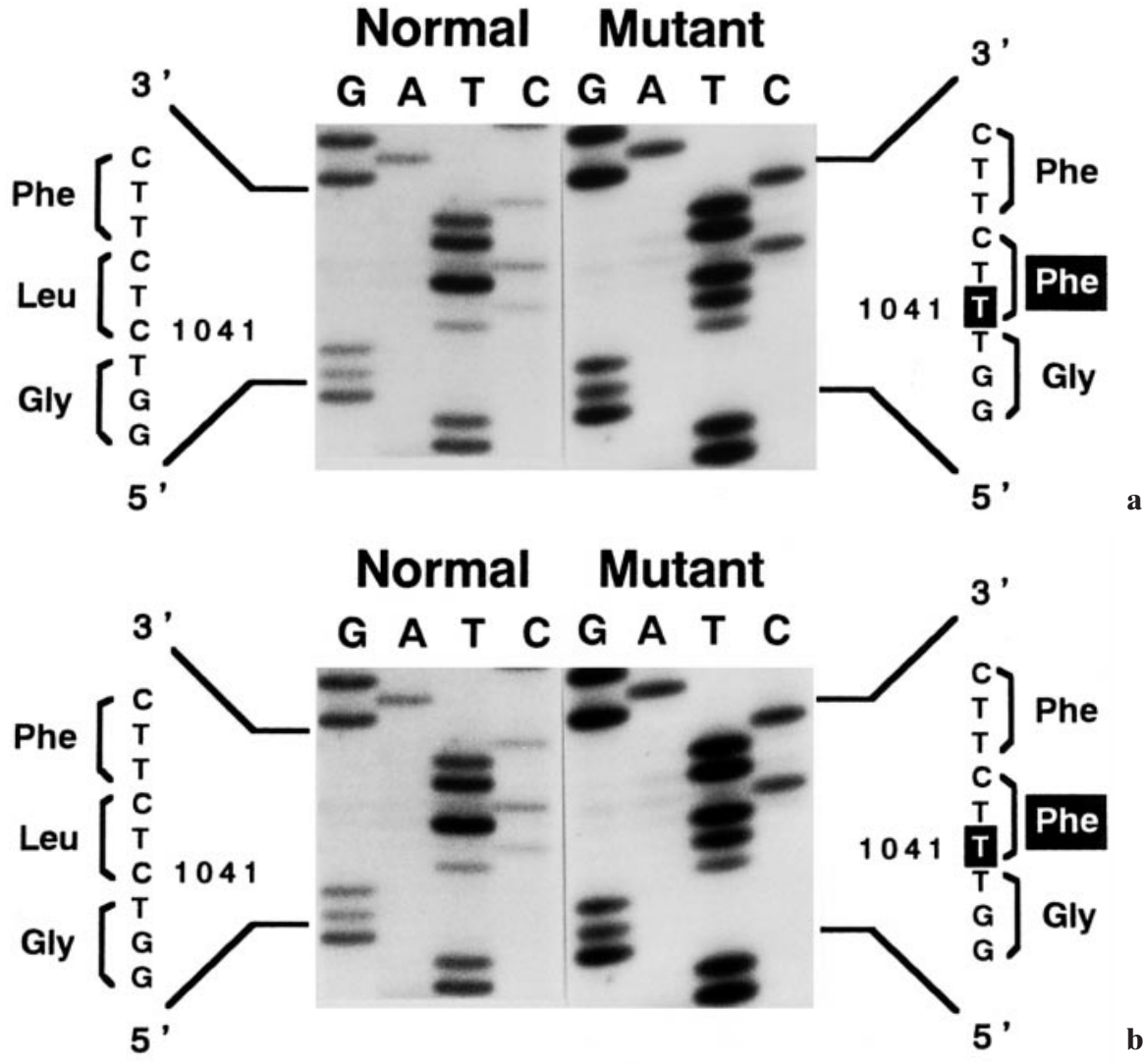

protein-coding region (open reading frame). By the quantitative PCR method, the relative amount of the patient's TNSALP mRNA after normalization by the internal control appeared to be almost equal to that in normal individuals (data not shown).

The amplified segment was inserted into a plasmid vector, and the coding sequences from two separated clones were compared to that of normal TNSALP. Two point mutations and one deletion were identified. A $\mathrm{T} \rightarrow \mathrm{C}$ transition at position 963 (963C) of the cDNA in exon 8 and a $\mathrm{C} \rightarrow \mathrm{T}$ transition at position 1041 (1041T) of the cDNA in exon 9 were found in a single clone (Fig. 1a). A deletion of $\mathrm{T}$ at 1735 (1735T) in exon 12 was detected in another clone (Fig. 1b).

To detect these mutations in the affected pedigree, we amplified the corresponding regions of genomic DNA from the proband's parents and then performed restriction fragment length polymorphism (RFLP) analysis. For PCRRFLP analysis of exons 9 and 12, we isolated intron 8, using the GeneAmp XL PCR Kit, and prepared primer SS (5'-GGGGAGCCTGCATTCCCTGA-3'), primer Ex-9A (5'-CACCAGCAAGAA-GCCTT-3'), primer Ex-12S (5'CTCACAACAACTACCAGGCG-3'), and primer A. The mutation $1041 \mathrm{~T}$ destroyed an Alw26I site, while the deletion $1735 \mathrm{~T}$ was expected to introduce a new $A v a \mathrm{I}$ site. The results of PCR-RFLP indicated that the proband had a compound heterozygote and had inherited the mutation $1041 \mathrm{~T}$ from her father and the deletion $1735 \mathrm{~T}$ from her mother (Fig. 2, RFLP data not shown). With regard to the mutation 1041T, 100 unrelated healthy Japanese individuals

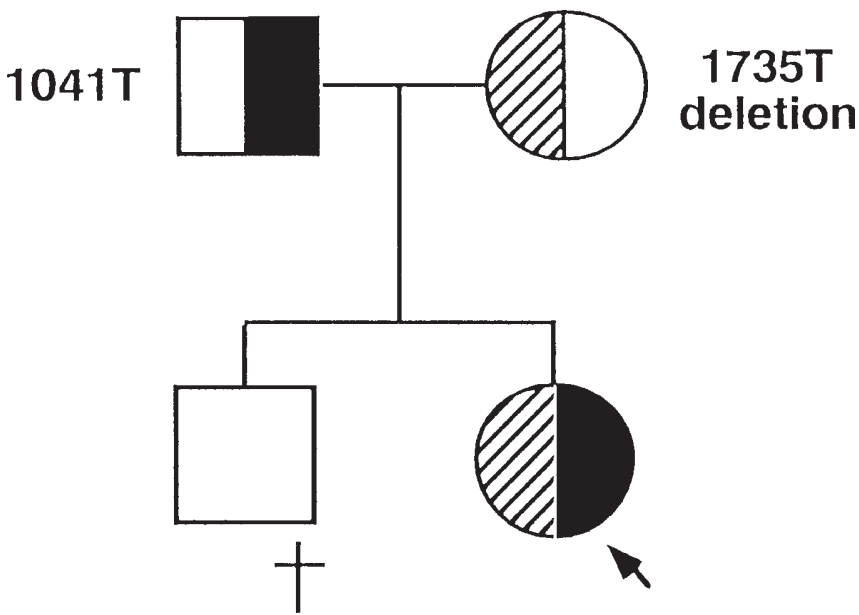

Fig. 2 The affected pedigree. The proband is indicated by the arrow. Solid filled symbols indicate the presence of the 1401T allele, and the symbols filled with diagonal lines represent the 1735T deletion allele

were investigated with PCR-RFLP and the mutation in question was not found.

To verify that the mutation $1041 \mathrm{~T}$ was responsible for the deficient activity of TNSALP observed in the proband, we purified mutation $1041 \mathrm{~T}$ cDNA and tested its ability to express ALP activity. NIH/3T3 cells transfected with the wild-type cDNA plasmid expressed ALP activity. In contrast, cells transfected with the mutant plasmid expressed half the level of ALP activity (Fig. 3). We obtained similar results by microscopic examination. 


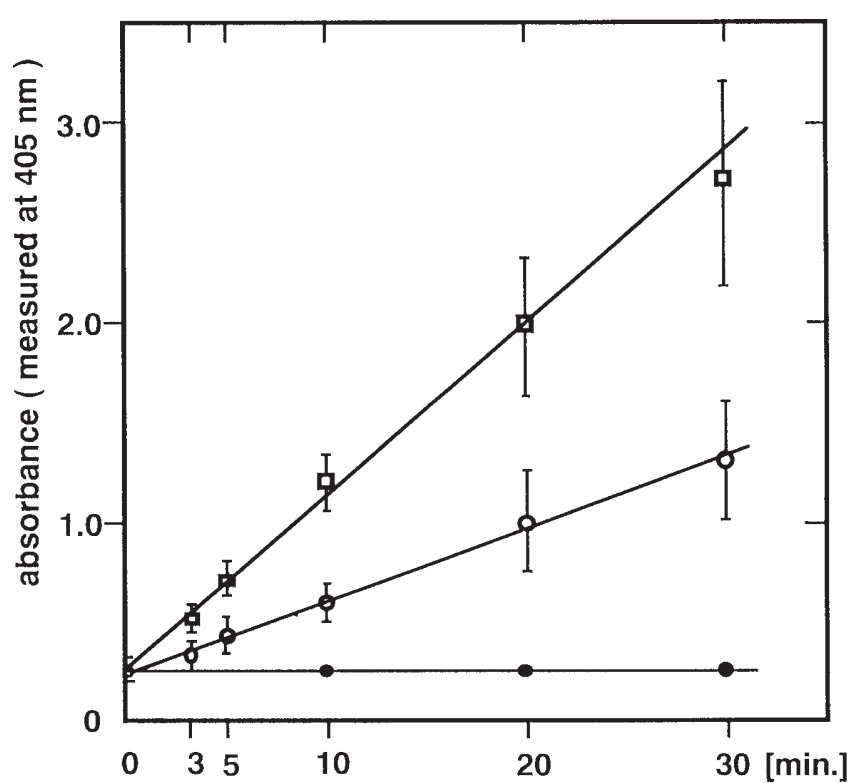

Fig. 3 Expression of the wild-type (squares) and mutant (circles) TNSALP cDNA. The mutant cDNA contains the $\mathrm{T} \rightarrow \mathrm{C}$ and $\mathrm{C} \rightarrow \mathrm{T}$ transitions at cDNA position 963 and 1041. For mock transfection (dots), no cDNA was used. ALP activity was measured by the method of Lowry et al. (1954), using a modification of the Committee on Enzyme of the Scandinavian Society for Clinical Chemistry and Clinical Physiology (1974). Cells transfected with the mutant plasmid expressed half the ALP activity expressed by the wild-type cDNA plasmid. Bars show SEM

\section{Discussion}

To date, in American, Canadian, and Japanese kindreds, 14 missense mutations and 1 frameshift mutation have been identified in the TNSALP gene (Fedde et al. 1996; Greenberg et al. 1993; Henthorn et al. 1992; Orimo et al. 1994; Ozono et al. 1996; Weiss et al. 1988).

In this study, we identified two missense mutations, $1041 \mathrm{~T}$ and $963 \mathrm{C}$, and a frameshift mutation (deletion 1735 T) in the TNSALP gene of a Japanese female patient with hypophosphatasia.

This is the first report of mutation 1041T, while mutation 963C has already been confirmed as a polymorphism (Henthorn et al. 1992). Mutation 1041T results in an amino acid change from Leu to Phe at codon 272 and destroys the Alw26I site. PCR-RFLP analysis of the parents' genomic DNA revealed the mutation in the patient's father. This missense mutation was shown by transfection analysis to reduce the activity of ALP by $50 \%$. These data were compatible with that of the father's serum ALP activity.

To further analyze this mutation, we compared it with the other ALP protein sequences available: those of Eschericia coli (Chang et al. 1986), Escherichia fergusoni, Serratia marcescens (DuBose and Hartl 1990), Drosophila melanogaster (Gene Bank data), Silk moth (Itoh et al. 1991), Gallus gallus (Crawford et al. 1995), mouse (Manes et al. 1990), rat (Misumi et al. 1988), bovine (Garattini et al. 1987), PALP (Millan 1986), and IALP (Berger et al. 1987).
The residue substitution by the novel mutation, Leu-272, was found not to be conserved in E. coli, E. fergusoni, or $S$. marcescens, by pairwise comparison using homology analysis. The residue is situated far from a group of metal ligand sites and from the catalytically important site, which was predicted from three-dimensional structure data of $E$. coli ALP (Sowadski et al. 1985). However, the residues surrounding Leu-272 are highly conserved in D. melanogaster to mammals (data not shown). These data suggest that the fragments of ambient Leu-272 constitute a significant domain and that the residue Leu-272 may play an important role in ALP activity.

The 1735T deletion mutation was identical to that reported by Orimo et al. (1994). Our PCR-RFLP analysis of the parents' genomic DNA revealed this mutation in the patient's mother. The deletion $1735 \mathrm{~T}$ causes a frameshift mutation downstream from the Leu at codon 503 and results in an incorrect translation of polypeptide owing to the frameshift, yielding a molecule that is about 80 amino acids longer than regular ALP. The ALP with this long amino acid chain influences the hydrophobic domain of the carboxyl-terminal sequence. Therefore, $\mathrm{COOH}$-terminal processing seems to be affected by this mutation, because it requires a short hydrophobic peptide at the carboxy-terminal and specific amino acids for correct processing (Kodukula et al. 1993). In the present study, a transfection analysis of this mutation was not performed. However, it seemed that serum ALP was more affected by the 1735T deletion than by the 1041C mutation, based on the mother's biochemical findings and on the detection of a lethal form of hypophosphatasia.

To our knowledge, there have been no reports on liver dysfunction in hypophosphatasia, although alkaline phosphatase is known to be a liver enzyme. In this pedigree, the proband and her brother died of hepatic failure at 16 years and 14 years of age, respectively. The details of these hepatic failures, however, remain unknown, since no pathology or biochemical examinations were perfomed on these patients. The biological function of ALP is little known despite the more than four decades of research on this protein. The only possible exception to this dearth of knowledge is in the area of hypophosphatasia.

In conclusion, we found a novel missense mutation in the TNSALP gene, which causes hypophosphatasia. Examination of its expression revealed that the mutation halved the level of ALP activity.

Acknowledgments Thanks to Dr. Momoi (Department of Pediatrics, Jichi Medical School) for instructive discussion. Thanks to Dr. Ookawara and Dr. Sakamoto (Department of Anatomy, Jichi Medical School) for the microscopic examination.

\section{References}

Amthauer R, Kudukula K, Brink L, Udenfriend S (1992) Phosphatidylinositol-glycan (PI-G) -anchored membrane proteins: Requirement of ATP and GTP for translation-independent $\mathrm{COOH}-$ terminal processing. Proc Natl Acad Sci USA 89: 6124-6128 
Berger J, Garattini E, Hua JC, Udenfriend S (1987) Cloning and sequencing of human intestinal alkaline phosphatase cDNA. Proc Natl Acad Sci USA 84: 695-698

Chang CN, Kuang WJ, Chen EY (1986) Nucleotide sequence of the alkaline phosphatase gene of Escherichia coli. Gene 44: 121-125

The Committee on Enzymes of the Scandinavian Society for Clinical Chemistry and Clinical Physiology (1974) Recommended methods for the determination of four enzymes in blood. Scand J Clin Lab Invest 33: 291-306

Coyne KE, Crisci A, Lublint DM (1993) Construction of synthetic signals for glycosyl-phosphatidylinositol anchor attatchment. J Biol Chem 268: 6689-6693

Crawford K, Weissig H, Binette F, Millan JL, Goetinck PF (1995) Tissue-nonspecific alkaline phosphatase participates in the establishment and growth of feather germs in embryonic chick skin cultures. Dev Dyn 204: 8-56

Currarino G, Neuhauser EBD, Reyesbach GC, Sobel EH (1957) Hypophosphatasia. Am J Roentgenol 78: 392-419

DuBose RF, Hartl DL (1990) The molecular evolution of bacterial alkaline phosphatase: Correlating variation among enteric bacteria of experimental manipulations of the protein. Mol Biol Evol 7: 547577

Fedde KN, Michell MP, Henthorn PS, Whyte MP (1996) Aberrant properties of alkaline phosphatase in patient fibroblasts correlate with clinical expressivity in severe forms of hypophosphatasia. J Clin Endocrinol Metab 81: 2587-2594

Fraser D (1957) Hypophosphatasia. Am J Med 22: 730-746

Garattini E, Hua JC, Udenfriend S (1987) Cloning and sequencing of bovine kidney alkaline phosphatase cDNA. Gene 59: 41-46

Greenberg CR, Taylor CLD, Haworth JC, Seargeant LE, Philipps S, Triggs-Rainen B, Chodirker BN (1993) A homoallelic Gly317 $\rightarrow$ Asp mutation in ALPL causes the perinatal (lethal) form of hypophosphatasia in Canadian Mennonites. Genomics 17: 215-217

Griffin CA, Smith M, Hentheron PS, Harris H, Weiss M, Rauducha M, Emanuel BS (1987) Human placental and intestinal alkaline phosphatase genes map to 2q34-q37. Am J Hum Genet 41: 1025-1034

Harris H (1989) The human alkaline phosphatase: What we know and what we don't know. Clin Chim Acta 186: 133-150

Henthorn PS, Raducha M, Fedde KN, Lafferty MA, Whyte MP (1992) Different missense mutation at the tissue-nonspecific alkaline phosphatase gene locus in autosomal recessively inherited forms of mild and severe hypophosphatasia. Proc Natl Acad Sci USA 89: 9924 9928

Itoh M, Takeda S, Yamamoto H, Izumi S, Tomino S, Eguchi M (1991) Cloning and sequence analysis of membrane-bound alkaline phosphatase cDNA of the silkworm, Bombyx mori Biochim Biophys Acta 1129: 135-138

Kodukula K, Gerber LD, Amthauer R, Brink L, Udenfriend S (1993) Biosynthesis of glycosylphosphatidylinositol (GPI)-anchored mem- brane proteins in intact cells: Specific amino acid requirements adjacent to the site of cleavage and GPI attachment. J Cell Biol 120: 657664

Lowry OH, Robertson NR, Wu ML, HIxon WS, Crawfold EJ (1954) The quantative histochemistry of brain II enzyme measurements. $J$ Biol Chem 207: 19-37

Manes T, Glade K, Ziomek CA, Millan JL (1990) Genomic structure and comparison of mouse tissue-specific alkaline phosphatase genes. Genomics 8: 541-554

McComb RB, Bowers GN, Posen S (1979) Alkaline phosphatase. Plenum, New York, Chapter 8: 373-524

Millan JL (1986) Molecular cloning and sequence analysis of human placental alkaline phosphatase. J Biol Chem 261: 3112-3115

Misumi Y, Tashiro K, Hattori M, Sakaki Y, Ikehara Y (1988) Primary structure of rat liver alkaline phosphatase deduced from its cDNA. Biochem J 249: 661-668

Nanba K, Aoki J, Sasaki N (1987) A new enzyme immuno-histochemical technique using alkaline phosphatase-labeled avidin and new fuchsin. Byouri To Rinnsyou 5: 333-339

Orimo H, Hayashi Z, Watanabe A, Hirayama T, Hirayama T, Shimada T (1994) Novel missense and frameshift mutations in the tissue-nonspecific alkaline phosphatase gene in Japanese patient with hypophosphatasia. Hum Mol Genet 3: 1683-1684

Ozono K, Yamagata M, Michigami T, Nakajima S, Sakai N, Cai G, Satomura K, Yasui N, Okada S, Nakayama M (1996) Identification of novel missense mutation (Phe310Leu and Gly439Arg) in a neonatal case of hypophosphatasia. J Clin Endocrinol Metab 44: 686-694

Sanger F, Nicklen S, Coulson AR (1977) DNA sequencing with chainterminating inhibitors. Proc Natl Acad Sci USA 74: 5463-5467

Sato N, Takahashi Y, Asano S (1994) Preferential usage of the bonetype leader sequence for the transcripts of liver/bone/kidney-type alkaline phosphatase gene in neutrophilic granulocytes. Blood 83: 1093-1101

Sowadski JM, Handschumacher MD, Murthy HMK, Foster BA, Wyckoff HW (1985) Refined structure of alkaline phosphatase from Escherichia at $2.8 \AA$ resolution. J Mol Biol 186: 417-733

Smith M, Weiss MJ, Griffin CA, Murray JC, Buetow KH, Manuel BS, Henthron PS, Harris H (1988) Region assignment of the gene for human liver/bone/kidney alkaline phosphatase to chromosome 1p36-p34. Genomics 2: 139-143

Weiss MJ, Henthorn PS, Lafferty MA, Slaughter C, Raducha M, Harris $\mathrm{H}$ (1986) Isolation and characterization of a cDNA encoding a human liver/bone/kidney alkaline phosphatase. Proc Natl Acad Sci USA 83: 7182-7186

Weiss MJ, Cole DEC, Ray K, Whyte MP, Lafferty MA, Mulivor RA, Harris H (1988) A missense mutation in the human liver/bone/ kidney alkaline phosphatase gene causing a lethal form of hypo-phosphatasia. Proc Natl Acad Sci USA 85: 7666-7669 